\title{
VERBAL FACTORS AS DETERMINANTS OF INTERMEDIATE-SIZE TRANSPOSITION IN PRESGHOOL CHILDREN
}

\author{
NORIHIKO KITAO',2 \\ Osaka Kyöiku University
}

\begin{abstract}
In Experiment I, 80 preschool children were divided into Concept and NonConcept groups according to a preliminary test, and further sub-divided into either Labeling or Non-Labeling group. In Experiment II, 100 preschool children in a concept group were divided into 5 groups as to the labeling in pretraining and the verbalization in original learning (OL) and test. They were then given intermediate-size transposition problem. The results were as follows: First, the possession of the concept "medium" facilitated transposition. Second, the labeling resulted in increased transposed responses in both Concept and Non-Concept groups. Finally, facilitated transposed responses were obtained with the verbalization both in OL and in the test, but not with that either in OL or in the test.
\end{abstract}

Reese (1966) reported that some instruction such as "medium" and "not bit and not little" increased the frequency of transposition in intermediate-size problems. According to Marsh and Sherman (1966), children who verbalized the relevant dimension (size) produced more transposed responses than those who verbalized the irrelevant dimension (color) in two-stimulus problems. However, these investigators seem to have paid little attention to children's concept knowledge. Therefore, it may be questioned whether verbal training such as instruction and verbalization provide a stimulus with a verbal label, or facilitate to use a verbal label which the subject has possessed. In the first experiment to be reported here, therefore, an attempt is made to compare children who possess appropriate verbal concepts with those who do not, concerning

\footnotetext{
1 The author is indebted to Dr. Akio Ono for editing the English phraseology of the manuscript and to Miss Yoshiko Hata for her assistance in collecting and analyzing the present data.

${ }^{2}$ Request for reprints should be sent to Norihiko Kitao, Department of Psychology, Osaka Kyöiku University, Tennöji-ku, Osaka, 543, Japan.
}

the effect of labeling pretraining to associate the relevant label with the stimulus.

On the other hand, according to Reese's mediational deficiency hypothesis (1962), it would be reasonable to assume that preschool children may not solve transposition problem on a verbal basis, even though they possess verbal concept. These children would benefit from additional training to verbalize the label together with choice responses, for mediational deficiency would be due to the disintegration between the verbal responses and the choice responses. Therefore, the second experiment is designed to examine the effect of the additional training to verbalize the label in original learning and/or transposition test-phase upon the performance of transposed responses.

\section{EXPERIMENT I}

\section{Method}

Design and Subjects. The experimental design was a $2 \times 2 \times 2 \times 2$ factorial. Two of them were between-subject factors, children's concept knowledge (Concept vs. Non-Concept) and 
labeling pretraining (Labeling vs. Non-Labeling). The others were within-subject factors, test set (Near test vs. Far test) and testing order (First test vs. Second test). The $S \mathrm{~s}$ were 80 kindergarten children, 40 Concept and 40 Non-Concept children, who were chosen from a pool of 89 children according to the performance of preliminary test. They were 37 boys and 43 girls, ranging in age from 57 to 70 months. The other two Concept children were randomly omitted from the experiment to equate the number of $S$ s between Concept and Non-Concept group, and the additional seven children (one Concept child and six Non-Concept children) were omitted because of failing to solve the original learning task within the allowable trials. A half of the $S \mathrm{~s}$ in each group was randomly assigned to either Labeling group or Non-Labeling group, resulting in belonging to each of the following four groups; Concept-Labeling (C-L), Concept-Non-Labeling (C-NL), Non-Concept-Labeling (NC-L) and Non-Concept-Non-Labeling (NC-NL). The average ages of these four groups were respectively $62.9,63.3,61.9$ and 61.3 months, having no significant differences with $\mathrm{F}(3,79)<1$.

Materials. The stimuli for a preliminary test were three green-colored pencils, 5.0, 7.5 and $10.0 \mathrm{~cm}$ in length. The stimuli for a main experiment were six red-colored squares different in size and numbered from 1 to 6 in the order of size. The smallest square, 1, had the area of 11.56 and the succeeding squares respectively increased in area with the ratio of $1: 1.4$. By combining these stimuli, three sets were constructed : 4-5-6 for the labeling and original learning, 3-4-5 for Near test and 1-2-3 for Far test. The stimuli were mounted on $12.5 \times 30 \mathrm{~cm}$ white cards, and horizontally arranged $2.5 \mathrm{~cm}$ apart each other in random order.

Procedure. The experiment consisted of four phases. In the first phase, the preliminary test, the $E$ placed three pencils on a table, and asked the $S$ as follows: "This pencil is large, isn't it? (pointing the largest). This pencil is small, isn't it? (pointing the smallest). Then, how large is this (pointing the medium)?"
(Question 1). If the $S$ was unable to answer correctly, he was given an additional question, "Which is a pencil of the intermediate size?", and then the main question mentioned above was repeated (Question 2). The $S$ who emitted an appropriate word, such as "middle" to either Question 1 or 2 was assigned to Concept group, and the other to Non-Concept group. Immediately after the preliminary test in the phase 2 (labeling pretraining), the $S$ s in Labeling groups were required to learn the verbal labels, "small ", " middle" and "large" for the stimuli 4,5 and 6 , respectively with the criterion of consecutive three perfect trials. In the phase 3 (original learning), all $S$ s were given a simultaneous discrimination task with Set 4-5-6, and the choice of 5 was rewarded. The non-correction procedure was used with the verbal reinforcement ("good" or " bad"). The original learning lasted to a criterion of 10 consecutive correct responses with a maximum of 36 trials. In the last phase (transposition test) they were given Near and Far tests in counterbalanced order, a half of them in each group receiving Near test first and the other Far test first. Each test consisted of 12 trials which were all non-rewarded. Throughout this experiment, the stimulus cards were manually presented depending upon the $S$ 's response rate.

\section{Results}

Labeling pretraining. Most of the $S \mathrm{~s}$ learned the verbal labels within about three trials excluding the criterion trials. The proportion of the $S \mathrm{~s}$ reaching the criterion on the first trial were 95 percent for Group C-L and 65 percent for Group NC-L. The difference between the groups was signifcant $\left(\chi^{2}=5.91, d f=1, p<.05\right)$.

Original learning. The number of trials to the criterion excluding the criterion trials were transformed to $\sqrt{x+.5}$ scores for the purpose of statistical analysis. Group means of these scores were 3.29 in Group C-L, 3.45 in Group C-NL, 3.17 in Group NC-L and 3.94 in Group NC-NL. The analysis of variance indicated that neither main effects nor in- 
TABLE 1

Mean number of transposed responses during 12 test trials as a function of concept knowledge, labeling, distance and testing order (Exp. I)

\begin{tabular}{|c|c|c|c|c|c|c|}
\hline \multirow{2}{*}{ Group } & \multirow{2}{*}{$\begin{array}{l}\text { No. } \\
\text { of } \\
S_{s}\end{array}$} & \multicolumn{2}{|c|}{ Near } & \multicolumn{2}{|r|}{ Far } & \multirow{2}{*}{ All } \\
\hline & & First & Second & First & Second & \\
\hline G-L & 20 & 8.7 & 8.5 & 6.8 & 7.6 & 7.9 \\
\hline $\mathrm{C}-\mathrm{NL}$ & 20 & 7.5 & 6.1 & 6.8 & 5.0 & 6.4 \\
\hline $\mathrm{NC}-\mathrm{L}$ & 20 & 8.9 & 8.1 & 5.6 & 3.1 & 6.4 \\
\hline NC-NL & 20 & 5.7 & 3.8 & 2.7 & 2.6 & 3.7 \\
\hline
\end{tabular}

teractions were statistically significant (Labeling: $F(1,72)=2.65, p>.05$; Concept $\times$ Testing order: $F(1,72)=1.43, p>.05$; others: $F<1$ ).

Transposition test. Table 1 presents the mean number of transposed responses in 12 test trials. An extension of the Lindquist (1956) Type IV design, used for the analysis of variance of these data, indicated that Concept groups marked transposed responses more than NonConcept groups $(F(1,72)=11.49, p<.01)$, that Labeling training inceased transposed responses $(F(1,72)=12.34, p<.01)$, and that there were more transposition in Near test than in Far test $(F(1,72)=38.69$, $p<.01)$, and in First test than in Second test $(F(1,72)=8.26, p<.01)$. A Concept by Distance interaction was also significant $(F(1,72)=8.51, p<.01)$, but the other interactions were not significant (Labeling $\times$ Distance: $\quad F(1,72)=3.55, p>.05$; others: $F<1$ ). The significant interaction reflects, as can be seen in Fig. 1, the

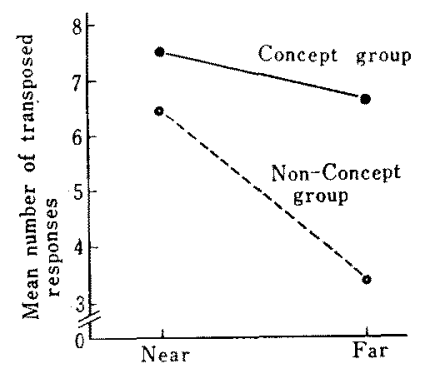

Fig. 1. Distance effect in transposotion as a function of concept knowledge (Exp. I). finding that the distance effect in transposition was larger in Non-Concept group $(p<.01)$ than in Concept group $(p<.05)$.

\section{EXPERIMENT II}

\section{Method}

Design and Subjects. The design of Exp. II was a 5 (Additional training) $\times 2$ (Distance) $\times 2$ (Testing order) factorial. The first factor was a between-subject factor, and yielded five groups: Labeling in pretraining (Group L), Labeling plus Verbalization in original learning (Group VO), Labeling plus Verbalization in test (Group VT), Labeling plus Verbalization in both original learning and test (Group VOT) and Control having no both Labeling and Verbalization (Group C). The second and third factors were the same within-subject factors as those in Exp. I. The $S$ s in Exp. II were 100 children (46 males and 54 females) from the same kindergarten as mentioned in Exp. I. Their age ranged from 58 to 70 months. They were all derived from a concept group according to the preliminary test, and randomly assigned to five groups in equal number (Group $\mathrm{L}$ and $\mathrm{C}$ were the identical to Group C-L and C-NL in Exp. I). The average ages in five groups were 62.7, 62.9, $63.3,63.7$ months respectively, showing no significant differences $(F(4,99)<1)$. Four $S$ s were omitted from this experiment because of the failure in original learning.

Materials. The stimulus for a preliminary test and main experiment were the same as used in Exp. I.

Procedure. The procedure was exactly the same as in Exp. I except that the $S$ s in Group VO, VT and VOT were required to verbalize a verbal label of the stimulus which $S$ chose just before choice responses in the phase mentioned previously.

\section{Results}

Labeling pretraining. All $S$ s learned the verbal labels within one or two trials. The proportion of the $S$ s reaching the criterion on the first trial were 95, 95, 100 and 100 percent for Group L, VO, VT 
TABLE 2

Mean number of transposed responses during 12 test trials as a function of training, distance and testing order (Exp. II)

\begin{tabular}{lrrrrrrr}
\hline \multirow{2}{*}{ Group } & $\begin{array}{r}\text { No. } \\
\text { of }\end{array}$ & \multicolumn{2}{c}{ Near } & \multicolumn{2}{c}{ Far } & All \\
& \multicolumn{1}{c}{$S$ s } & First & Second & First & Second & \\
\cline { 1 - 4 } L & 20 & 8.7 & 8.5 & 6.8 & 7.6 & 7.9 \\
VO & 20 & 8.1 & 6.4 & 6.3 & 7.2 & 7.0 \\
VT & 20 & 8.2 & 6.8 & 5.4 & 7.0 & 6.9 \\
VOT & 20 & 10.8 & 11.3 & 9.4 & 10.2 & 10.4 \\
C & 20 & 7.5 & 6.1 & 6.8 & 5.0 & 6.4
\end{tabular}

and VOT respectively.

Original learning. Mean number of trials ( $\sqrt{x+.5}$ transformed scores) to the criterion excluding the criterion trials were $3.29,2.84,2.80,2.54$ and 3.45 for Group $\mathrm{L}, \mathrm{VO}, \mathrm{VT}, \mathrm{VOT}$ and $\mathrm{C}$ respectively. The difference between the groups were not significant $(F(4,90)=2.00, p>.05)$. It was found, in the present case, that the verbalization of the relevant label did not facilitate to solve the discrimination problem.

Transposition test. Table 2 presents the mean number of transposed responses. The analysis of variance of these data, using an extension of the Lindquist (1956) Type IV design, revealed that main effects of Additional training and Distance were significant at $1 \%$ level with $F(4,95)=$ 5.49 and $F(1,90)=13.96$ respectively, and that neither the main effect of Testing order nor the interaction were significant $(F<1)$. Comparison between Additional training conditions, using Scheffé method, indicated that Group VOT made more transposed responses than Group VO $(p<.05)$, Group VT $(p<.05)$ and Group $\mathrm{C}(p<.01)$, and that the differences of transposed responses between the two groups of latter three were not significant.

Further examination was performed on the responses made in the test phase of Group VT and VOT. Table 3 shows the number of Non-Mediated Responses (NMR) which were the incorrect choice responses (pointing a large or a small
TABLE 3

Number of non-mediated responses in transposition tests (Exp. II)

\begin{tabular}{lrrrrrrr}
\hline \multirow{2}{*}{ Group } & \multicolumn{2}{c}{ Near } & & \multicolumn{2}{c}{ Far } & \multirow{2}{*}{ All } \\
\cline { 2 - 3 } & First & Second & & First & Second & \\
\hline VT & 10 & 8 & & 23 & 14 & 55 \\
VOT & 5 & 2 & & 16 & 4 & 27
\end{tabular}

square) accompanying with a correct verbalization ("medium"). As seen in the table, Group VT made approximately two times as many NMR as those of Group VOT, and they made more NMR in Far test than those in Near test.

\section{Discussion}

One of the findings of Experiment I was that the labeling pretraining increased transposed responses in both Concept and Non-Concept groups. This finding would preclude a choice between the alternatives previously mentioned, leading to the speculation that the labeling pretraining has dual functions, i.e. providing a stimulus with a verbal label and facilitating to made use of a verbal label which the subject has possessed. In the present case, however, some children in Non-Concept group were able to point out the "medium", even though they could not verbalize the label. It may be, therefore, that the former function would be in action only among children who can implicitly understand the label provided by the experimenter.

The other finding in Experiment I was that the possession of concept knowledge facilitated transposition, and this effect was larger in Far test than in Near test. This fact seems to support Kuenne's (1946) hypothesis in which the incidence of transposition should differ depending upon the child's linguistic competence.

In Experiment II, it was assumed that the verbalization would facilitate transposition and overcome the mediational deficiency observed in Experiment I. Our results indicated that the verbalization in 
both original learning and test facilitated transposition, but that the verbalization in either original learning or test did not. The former fact seems to support the present prediction drawn from the verbal mediation hypothesis, but the latter, which is an unexpected outcome, would be rather along the line of the discriminability hypothesis (Stevenson \& Bitterman, 1955). That is, the verbalization in either original learning or testing phase would decrease transposed responses because it increased the difference between the two situations. Therefore, if this unexpected factor is excluded in some way, then the verbalization should facilitate verbal mediation, and consequently increase transposed responses.

In addition, it was found in Experiment II that Group VOT made fewer NMR than those of Group VT. This finding indicates that the verbalization in original learning had the effect to increase the verbal control over motor response in transposition problem. Furthermore, it suggests that most of meditaional deficiencies observed in young children's transposition behavior is the failure of a potential mediator to control behavior, which was termed as "control deficiency" by Kendler (1972).

\section{REFERENCE}

KendeER, T.S. 1972 An ontogeny of mediational deficiency. Child Development, 43, 117.

Kuenne, M.R. 1946 Experimental investigation of transposition behavior in young children. Fournal of Experimental Psychology, 36, 471-490.

LindQuist, E. F. 1956 Design and analysis of experiment in psychology and education. Boston: Houghton Mifflin.

Marsh, G., \& Sherman, M. 1966 Verbal mediation of transposition as a function of age level Journal of Experimental Child Psychology, 4, 90-98.

ReESE, H. W. 1962 Verbal mediation as a function of age level. Psychological Bulletin, 59, 502-509.

ReEse, H.W. 1966 Verbal effect in the intermediate-size transposition problem. Foumal of Experimental Child Psychology, 3, 123-130.

Stevenson, H.W., \& BitTerman, M. E. 1955 The distance effect in the transposition of intermediate size by children. American Journal of Psychology, 68, 274-279.

(Received June 13, 1974) 